\title{
Benefícios da oximetria de pulso na triagem neonatal para detectar cardiopatias congênitas
}

Benefits of pulse oximetry in neonatal screening to detect congenital heart diseases

Beneficios de la pulsioximetría en el cribado neonatal para detectar las enfermedades cardíacas congénitas

\section{RESUMO}

OBJETIVO: Avaliar a efetividade da oximetria de pulso na triagem neonatal a fim de facilitar o diagnóstico de cardiopatias congênitas bem como observar as mais sensíveis ao teste. MÉTODO: Trata-se de uma revisão sistemática da literatura que incluiu artigos publicados entre 2014 e 2018. Os descritores escolhidos foram inseridos nas bases de dados PubMed e MedLine. RESULTADOS: A busca resultou em 34 artigos, sendo avaliados 5 artigos que respeitavam os critérios de exclusão e inclusão estabelecidos. Com base nos resultados desses estudos, a acurácia da oximetria foi avaliada de acordo com a sensibilidade que variou de 60 a 100\% e a especificidade que girou em torno de 94 a 99,9\%. CONCLUSÕES: A oximetria evidenciou papel importante no processo que antecede o diagnóstico das cardiopatias congênitas ao permitir assistência e intervenção cirúrgica precoce nos casos que demandam correção urgente do defeito cardíaco.

DESCRITORES: Oximetria de pulso; Benefícios; Congênito; Rastreamento; Recém-nascidos.

\section{ABSTRACT}

OBJECTIVE: To evaluate the effectiveness of pulse oximetry in neonatal screening in order to facilitate the diagnosis of congenital heart diseases as well as to observe the most sensitive to the test. METHODS: This is a systematic literature review that included articles published between 2014 and 2018. The chosen keywords were entered into PubMed and MedLine databases. RESULTS: The search resulted in 34 articles, from which 5 articles that met the established exclusion and inclusion criteria were evaluated. Based on the results of these studies, the accuracy of oximetry was evaluated according to sensitivity, which ranged from 60 to $100 \%$, and specificity, which ranged from 94 to $99.9 \%$. CONCLUSIONS: Oximetry has shown an important role in the process preceding the diagnosis of congenital heart defects by allowing early assistance and surgical intervention in cases that require urgent correction of the heart defect.

DESCRIPTORS: Pulse Oximetry; Benefits; Congenital; Screening; Newborns.

\section{RESUMEN}

OBJETIVO: Evaluar la efectividad de la oximetría de pulso en el tría neonatal para facilitar el diagnóstico de las cardiopatías congénitas, así como observar las más sensibles a el test. MÉTODOS: Se trata de una revisión bibliografía sistemática que incluye artículos publicados entre 2014 y 2018. Los descriptores elegidos fueron insertados en las bases de datos PubMed y MedLine. RESULTADOS: La búsqueda dio lugar a 34 artículos, y fueron evaluados 5 artículos que cumplían los criterios de exclusión e inclusión establecidos. Basándose en los resultados de esos estudios, la precisión de la oximetría se evaluó según la sensibilidad, que osciló entre el 60 y el 100\%, y la especificidad, que osciló entre el 94 y el 99,9\%. CONCLUSIONES: La oximetría ha demostrado tener un papel importante en el proceso que precede al diagnóstico de los defectos cardíacos congénitos al permitir una asistencia e intervención quirúrgica precoz en los casos que requieren una corrección urgente del defecto cardíaco.

DESCRIPTORES: Oximetría de pulso; Beneficios; Congénito; Seguimiento; Recién nacidos.

RECEBIDO EM: 27/01/2021 APROVADO EM: 05/04/2021

\section{Isabella Ellen de Souza Gomes}

Estudante de medicina da Universidade Católica de Pernambuco.

ORCID: 0000-0002-3516-9370

\section{Maryna Morais de Andrade Cavalcanti}

Estudante de medicina da Universidade Católica de Pernambuco.

ORCID: 0000-0003-4657-8820 
Isabelly Stefany Silva de Paula

Estudante de medicina da Universidade Católica de Pernambuco. ORCID: 0000-0002-3319-6076

\section{Letícia Dowsley}

Estudante de medicina da Universidade Católica de Pernambuco.

ORCID: 0000-0002-4775-9888

\section{Luiza Maria Lopes Padilha}

Estudante de medicina da Universidade Católica de Pernambuco.

ORCID: 0000-0003-4201-8033

\section{Thainá de Oliveira Siqueira}

Estudante de medicina da Universidade Católica de Pernambuco.

ORCID: 0000-0001-5394-3705

\section{INTRODUÇÃO}

A s cardiopatias congênitas devem-se a alterações na estrutura do coração ou dos grandes vasos estando presentes desde o nascimento. Essas alterações representam cerca de $40 \%$ de todas as malformações congênitas e levam a uma grande taxa de morbimortalidade neonatal e infantil, sendo a segunda causa de morte em recém-nascidos com menos de um ano de vida ${ }^{1,2}$.

Segundo dados de 2018 da Secretaria do Ministério da Saúde a incidência dessas cardiopatias no Brasil é estimada em 1 a cada 100 nascidos vivos (1\%), ou seja, aproximadamente 30 mil crianças nascem no Brasil com essa patologia todos os anos $^{3}$. O número de óbitos relacionados às cardiopatias congênitas em 2015 no Brasil foi de 107 casos para cada 100 mil nascidos vivos, nos quais 30\% desses óbitos ocorreram no período neonatal precoce. Assim, quanto antes feita a detecção dessas malformações, maior a chance de tratar essas condiçôes rapidamente e desse modo reduzir a mortalidade $\mathrm{e}^{4}$.

A oximetria de pulso é um exame acessível, com resultado imediato e de fácil aplicabilidade que consiste na aplicação de um transdutor no pulso do membro superior direito e outro no tornozelo de qualquer dos membros inferiores do bebê. O resultado de ambas medidas da saturação são comparadas de forma que o exame é dito alterado quando a saturação é menor que $95 \%$ ou quando a diferença das
A oximetria

de pulso é um

exame acessível,

com resultado

imediato e de fácil

aplicabilidade que

consiste na aplicação

de um transdutor no

pulso do membro

superior direito e

outro no tornozelo

de qualquer dos

membros inferiores

do bebê. saturações dos membros é maior ou igual a 3\%. Após identificada e confirmada a alteração, o bebê necessita realizar o ecocardiograma, que é o exame mais eficaz para confirmação da cardiopatia 5 .

Dessa forma, esta revisão metodológica tem a finalidade de reunir resultados acerca da acurácia e dos benefícios da oximetria de pulso na detecção precoce das cardiopatias congênitas. Assim, pretende-se questionar a utilidade desse método e seus efeitos na diminuição da morbimortalidade provocada por essas patologias.

\section{OBJETIVO}

Avaliar a efetividade da oximetria de pulso na detecção de cardiopatias congênitas em neonatos. Através da identificação do número de recém-nascidos diagnosticados nas primeiras horas de vida durante a triagem. Bem como distinguir os tipos de cardiopatias congênitas que possuem maior sensibilidade ao exame e avaliar a repercussão do diagnóstico precoce de cardiopatias congênitas na determinação de um prognóstico favorável.

\section{MÉTODO}

Este estudo trata-se de uma revisão integrativa da literatura desenvolvida no período de agosto a setembro de 2018 . A pesquisa foi feita em duas bases de dados: Public Medicine Library (PubMed) e Medical Analysis Retrieval System Online (MedLine). Nessa pesquisa foram 
usados os descritores: oximetry pulse, newborns, benefits, congenital e screening com o operador booleano "AND".

$\mathrm{Na}$ busca em ambas plataformas foi utilizado o filtro referente ao desenho de estudo e incluídas revisões sistemáticas e artigos originais publicados entre $2014 \mathrm{e}$ 2018. Dessa forma, a busca resultou em 34 artigos dos quais foram analisados os títulos e resumos por dois autores de maneira independente. Os critérios escolhidos excluíram artigos duplicados e aqueles que não abordavam a pergunta norteadora ou o objetivo principal da pesquisa.

Por fim, foi realizada a leitura crítica dos textos na íntegra e analisados os dados científicos que serviram de base para a construção desse estudo. Quanto ao critério do idioma, as línguas inglesa e espanhola incluídas, restando 5 artigos na composição desta revisão de acordo com os critérios de elegibilidade adotados.

\section{RESULTADOS}

A maioria dos estudos foram feitos em maternidades nas quais o exame foi realizado em duas etapas. Quando a saturação da primeira etapa era baixa, isto é, menor ou igual a $95 \%$, os recém-nascidos eram submetidos novamente à medição do oxímetro. Aqueles que obtinham saturação anormal na segunda aferição passavam pelo ecocardiograma a fim de confirmar a patologia ${ }^{6,7}$.

Um estudo realizado na China contou com 6.750 recém-nascidos nos quais o oxímetro detectou anormalidade na saturação de 46 dos 49 casos assintomáticos de cardiopatias que necessitam de intervenção durante a infância. Também foram identificados os 8 casos assintomáticos de cardiopatias congênitas críticas as quais necessitam de intervenção nos primeiros 28 dias de vida. O oxímetro sozinho foi
A maioria dos estudos

foram feitos em

maternidades nas

quais o exame foi

realizado em duas

etapas. Quando a

saturação da primeira

etapa era baixa, isto é,

menor ou igual a 95\%,

os recém-nascidos

eram submetidos

novamente à medição

do oxímetro. Aqueles

que obtinham

saturação anormal

na segunda aferição

passavam pelo

ecocardiograma a

fim de confirmar a

patologia capaz de detectar $100 \%$ dos casos de estenose pulmonar crítica, tetralogia de Fallot e atresia pulmonar. Quando o oxímetro foi associado ao exame clínico houve acréscimo no percentual de detecção das seguintes cardiopatias: Truncus arteriosus ( $\uparrow 20 \%)$, ventrículo único ( $\uparrow \%)$, atresia pulmonar ( $\uparrow 7 \%)$, transposição das grandes artérias ( $\uparrow \% \%)$, dupla via de saída de ventrículo direito ( $\uparrow 33 \%)$, síndrome da hipoplasia do coração esquerdo ( $\uparrow 24 \%)$, coarctação de aorta $(\uparrow 14 \%)$, arco aórtico interrompido ( $\uparrow 40 \%)$ e drenagem anômala total das veias pulmonares $(\uparrow 47 \%)^{7}$.

Outra pesquisa realizada na Austrália observou a oximetria em 18.801 bebês no decorrer de um período de 42 meses. Houve alteração na triagem em 15 deles, destes, 4 foram confirmados posteriormente pelo ecocardiograma como cardiopatas (verdadeiros positivos). As cardiopatias identificadas foram: transposição de grandes artérias, estenose de aorta severa, drenagem anômala total das veias pulmonares e estenose pulmonar crítica ducto dependente. Os 6 falsos positivos com alteração na oximetria, possuíam alguma patologia de origem pulmonar ${ }^{6}$.

Na revisão de Engel e Kochilas ${ }^{8}$ é mencionado um protocolo que definiu 7 lesões primárias capazes de serem altamente diagnosticadas após a suspeição indicada pela oximetria, são elas: Síndrome da hipoplasia do coração esquerdo, atresia pulmonar, drenagem anômala total das veias pulmonares, transposição das grandes artérias, tetralogia de Fallot, atresia de tricúspide, truncus arteriosus, embora outras condições também sejam possivelmente triadas pelo exame ${ }^{8}$.

Desse modo, com base nos artigos avaliados, os resultados revelaram uma variação na sensibilidade da oximetria de 60 a $100 \%$ enquanto a especificidade variou em média de 94 a 99,9\%. Quanto à taxa de falsos positivos, os valores oscilaram de $0 \mathrm{a}$ $1,8 \%^{6,8,9,10}$. (Ver tabela 1 ).

O exame da oximetria de pulso apresentou modificações nas taxas de sensibilidade e especificidade a depender do tempo de vida em que foi realizado nos recém-nascidos. Houve redução da taxa de 
sensibilidade quando o exame foi feito antes de 24 horas e após esse período $(88,2 \%$ vs $78,4 \%)^{7,10}$. A taxa de especificidade foi de $99,4 \%$ antes de $24 \mathrm{~h}$ e $99,7 \%$ após $24 \mathrm{~h}$. A taxa de falsos positivos também sofreu modificações, sendo de $0,55 \%$ nas primeiras $24 \mathrm{~h}, 0,29 \%$ entre 25 e $48 \mathrm{~h}$ e $0,26 \%$ de 49 a $72 h^{7}$.

\section{DISCUSSÃO}

O diagnóstico das cardiopatias congênitas é feito por meio da observação criteriosa de alguns sinais clínicos, sendo os principais a cianose, a taquipneia e a presença de sopro cardíaco ${ }^{11}$. A elevada concentração de hemoglobina permite perceber a cianose somente quando a saturação de oxigênio for inferior a $80 \%$, sendo a hipóxia de difícil detecção no recém-nascido ${ }^{12}$.

Um dos principais papéis da oximetria está no reconhecimento dos 60 a 100\% dos recém-nascidos que possuem cardiopatias congênitas críticas. Estas se caracterizam por um defeito cardíaco específico em que $80 \%$ dos casos necessita de intervenção cirúrgica ou cateterismo e 50\%, que esses procedimentos sejam realizados no primeiro ano de vida ${ }^{13,14,15}$.

Em 2010, o Comitê Consultivo da Secretaria sobre Transtornos Hereditários em Recém-Nascidos definiu que as cardiopatias mais detectadas estão, sobretudo, entre 7 defeitos específicos tidos como alvos da triagem por oxímetro, são elas: síndrome da hipoplasia do coração esquerdo, atresia pulmonar, drenagem anômala total das veias pulmonares, transposição de grandes artérias, tetralogia de Fallot, atresia de tricúspide e truncus arteriosus8. Essas lesões, comumente associadas a hipoxemia, são causa de significativa morbimortalidade quando o diagnóstico é feito tardiamente em virtude da possibilidade de colapso circulatório antes da alta hospitalar ${ }^{8,16}$.

O exame da oximetria de pulso apresentou elevada acurácia na triagem de cardiopatias congênitas com altos valores de sensibilidade e especificidade, chegando a números próximos de $100 \%$, assim como baixos números de falsos positivos que não superaram a taxa de $2 \%$ 6,78,9, 10 .

Apesar do exame apresentar uma baixa taxa de falsos positivos, entre 35 a $74 \%$ deles foi possível detectar outras cardiopatias não críticas, infecções e o estágio inicial de distúrbios hormonais. Dessa forma, o oxímetro

\section{O diagnóstico}

das cardiopatias

congênitas é

feito por meio da

observação criteriosa

de alguns sinais

clínicos, sendo os

principais a cianose,

a taquipneia e a

presença de sopro

cardíaco. A elevada

concentração

de hemoglobina

permite perceber

a cianose somente

quando a saturação

de oxigênio for

inferior a $80 \%$, sendo

a hipóxia de difícil

detecção no recém-

nascido mostrou-se capaz de pressupor a presença de outras patologias além das cardíacas?

A justificativa para elevada acurácia da oximetria reside no fato de que determinadas cardiopatias apresentam graus de hipóxia no período neonatal em consequência da mistura de sangue da circulação sistêmica com a pulmonar, o que leva à redução da saturação periférica. A identificação da queda na saturação auxilia também no diagnóstico dos recém-nascidos assintomáticos e dessa forma viabiliza o tratamento da cardiopatia. A partir dessa alteração constatada pelo oxímetro os bebês são encaminhados para realizarem o ecocardiograma e em seguida serem avaliados por um especialista. Com base na detecção da hipóxia torna-se possível dar o diagnóstico precocemente, ainda nas primeiras $24 \mathrm{~h}^{1,5,12}$.

O mecanismo de origem da sintomatologia dessas cardiopatias se deve à transição da circulação fetal para a circulação neonatal que nesses casos estará alterada devido aos defeitos cardiovasculares 17. Foi observado que os níveis de saturação periférica podem sofrer modificações de acordo com o tempo de vida, pois o fechamento do canal arterial ocorre de 24 a 48 horas após o nascimento e a partir desse evento as manifestações clínicas se tornam mais evidentes ${ }^{16}$.

A taxa de sensibilidade se apresentou maior quando a triagem foi aplicada antes das $24 \mathrm{~h}$ de vida. No entanto, nos casos em que a oximetria foi realizada depois de $24 \mathrm{~h}$ houve um aumento significativo na taxa de especificidade com reduzida taxa de falsos-positivos. Entretanto, apesar desse ganho na especificidade, a demora na realização da oximetria torna possível o surgimento de sintomas graves nos quais $9 \%$ dos casos de cardiopatias congênitas críticas sofrem colapso circulatório ${ }^{\text {. }}$.

Assim, o diagnóstico precoce poderia evitar problemas decorrentes da evolução do quadro como descompensação hemodinâmica, choque e acidose que implicam a curto prazo em mau prognóstico como mortalidade peri-operatória, e a longo prazo em sequelas neurológicas ${ }^{12}$. 


\section{artigo}

Gomes, I.E.S.; Cavalcanti, M.M.A; Paula, I.S.S.; Dowsley, Li; Padilha, L.M.L.; Siqueira, R.O.

Benefícios da oximetria de pulso na triagem neonatal para detectar cardiopatias congênitas

\section{CONCLUSÃO}

$\mathrm{O}$ oxímetro de pulso usado na triagem dos neonatos se mostrou eficaz para auxiliar na detecção das cardiopatias congênitas causadoras de hipóxia. Isso porque a oximetria apresentou elevada acurácia na identificação não só das cardiopatias como também de outras afecções, especialmente quando o exame foi executado nas primeiras 24 horas de vida. O diagnóstico feito nas primeiras horas de vida possibilita ao neonato o tratamento adequado de maneira mais precoce e assim previne a piora do quadro.
Em razão da grande incidência e gravidade das cardiopatias congênitas, esses estudos que enfatizam o papel do oxímetro no diagnóstico precoce destas patologias devem servir como base para a promoção de protocolos que implementem o seu uso na triagem, sobretudo em locais onde a assistência pré-natal é precária.

\section{REFERÊNCIAS}

1. Ministério da Saúde (Brasil). Síntese de evidências para políticas de saúde: diagnóstico precoce de cardiopatias congênitas [internet]. 2018 [citado em: 9 out 2018]. Disponivel em: http://bvsms. saude.gov.br/bvs/publicacoes/sintese_evidencias_politicas_cardiopatias_cogenitas.pdf

2. Lopes SAVA, Guimarães ICB, Costa SFO, Acosta AX, Sandes KA, Mendes CMC. Mortalidade para Cardiopatias Congênitas e Fatores de Risco Associados em Recém-Nascidos. Um Estudo de Coorte [internet]. Sociedade Brasileira de Cardiologia. 2018 [citado em: 9 out 2018]. Disponível em: http://publicacoes.cardiol.br/portal/abc/ portugues/2018/v11105/mortalidade-para-cardiopatias-congenitas-e-fatores-de-risco-associados-em-recem-nascidos.asp

3. Frasão G. Espera por cirurgias de cardiopatia congênita infantil cai 35\% [internet]. Ministério da Saúde (Brasil). 2018 [citado em 10 set 2018]. Disponivel em: https://www-hmg.saude.gov.br/ noticias/agencia-saude/43527-em-um-ano-cirurgias-de-cardiopatia-congenita-infantil-crescem-8

4. Ministério da Saúde (Brasil), DATASUS. Sistema de Informações sobre Mortalidade - SIM. [internet] 2015 [citado em: 10 set 2018]. Disponível em: http://www2.datasus.gov.br/DATASUS/index.php?area $=060701$

5. Sociedade Brasileira de Pediatria (Brasil). Diagnóstico precoce de cardiopatia congênita crítica: oximetria de pulso como ferramenta de triagem neonatal. Sociedade Brasileira de Pediatria [internet]. 2011 [citado em 8 out 2018]. Disponivel em: http://www. sbp.com.br/fileadmin/user_upload/2015/02/diagnostico-precoce-oximetria.pdf

6. Bhola K, Kluckow M, Evans N. Post-implementation review of pulse oximetry screening of well newborns in an Australian tertiary maternity hospital. J Paediatr Child Health [internet]. 2014 [citado em 10 ago 2018]; 50(11):920-5. Disponivel em: https:// pubmed.ncbi.nlm.nih.gov/24923996/

7. Zhao QM, Ma XJ, Ge XL, Liu F, Yan WL, Wu L, et al. Pulse oximetry with clinical assessment to screen for congenital heart disease in neonates in China: a prospective study: Lancet [internet]. 2014 [citado em 12 set 2018];384(9945):747-54. Disponivel em: https://pubmed.ncbi.nlm.nih.gov/24768155/

8. Engel MS, Kochilas LK. Pulse oximetry screening: a review of diagnosing critical congenital heart disease in newborns. Med Devices (Auckl) [internet]. 2016 [acesso em 20 ago 2018]; Disponível em: https://www.ncbi.nlm.nih.gov/pmc/articles/PMC4946827/

9. Narayen IC, Blom NA, Ewer AK, Vento M, Manzoni P, te Pas AB. Aspects of pulse oximetry screening for critical congenital heart defects: when, how and why? Arch Dis Child Fetal Neonatal [inter- net]. 2016 [citado em 13 set 2018]; Disponível: https://pubmed. ncbi.nlm.nih.gov/26369369/

10. Plana MN, Zamora J, Suresh G, Fernandez-Pineda L, Thangaratinam S, Ewer AK. Pulse oximetry screening for critical congenital heart defects. Cochrane Database Syst Rev [internet]. 2018 [citado em 20 set 2018];3(3). Disponivel em: https://pubmed.ncbi. nlm.nih.gov/29494750/

11. Ministério da Saúde. Atenção à saúde do recém-nascido: Guia para os profissionais de saúde [internet]. 2012 [citado em 9 out 2018]. Disponível em: http://bvsms.saude.gov.br/bvs/publicacoes/atencao_saude_recem_nascido_profissionais_v3.pdf

12. Morais S, Mimoso G. Oximetria de pulso no diagnóstico de cardiopatia congênita. Sugestões para a implementação de uma estratégia de rastreio. Portuguese Journal of Pediatrics [internet]. 2014 [citado em 9 out 2018]. Disponivel em: https://revistas. rcaap.pt/app/article/view/2717/0

13. Castro MS. Teste de triagem neonatal de cardiopatias congênitas: Uma revisão Integrativa. [TCC na internet]. Universidade Federal Fluminense (UFF). 2016 [citado em: 9 out 2018]. Disponivel em: https://app.uff.br/riuff/bitstream/1/3549/1/TCC\%20 Mariana\%20da\%20Silva\%20Castro.pdf. Trabalho de Conclusão de Curso em Enfermagem e Licenciatura

14. Sociedade brasileira de pediatria. Tempo de permanência hospitalar do recém-nascido a termo saudável [internet]. 2015 [citado em: 9 out 2018]. Disponível em: http://www.sbp.com.br/fileadmin/user_upload/2015/02/doc_tempo-permanencia_rn.pdf

15. Ministério da Saúde (Brasil). Governo federal lança plano para ampliar atendimento de crianças com cardiopatias congênitas. [internet]. 2017. [citado em: 9 out 2018]. Disponivel em:http:// portalarquivos.saude.gov.br/images/pdf/2017/julho/11/21.06_ Cardiopatia\%20Congenita\%20pediatrica.pdf

16. Ministério da Saúde. Nota técnica N 7/2018-CGSCAM/DAPES/SAS/M [internet]. 2018 [citado em: 9 out 2018]. Disponível em: https://portalarquivos2.saude.gov.br/images/pdf/2018/junho/12/SEI-MS-2937931-Nota-Tecnica.pdf

17. Ferreira S. Exame cardiovascular do recém-nascido [internet]. Pedipedia. 2018 [citado em: 9 nov 2018]. Disponível em: http:// www.pedipedia.org/pro/artigo-profissional/exame-cardiovascular-do-recem-nascido

18. COREN - Conselho regional de enfermagem da Bahia (Brasil). Teste do Coraçãozinho realizado pela Equipe de Enfermagem [internet]. 2014 [citado em 8 nov 2018]. Disponível em: http://ba.corens.portalcofen.gov.br/parecer-coren-ban\%E2\%81\%BO-0102014_15502.html 